\title{
MaRGEE: Move and Rotate Google Earth Elements
}

\author{
Mladen M. Dordevic ${ }^{1}$ and Steven J. Whitmeyer ${ }^{1}$
}

Geology and Environmental Science

James Madison University, Harrisonburg, VA 22807

540-568-7119, fax: 540-568-8058

1dordevmx@jmu.edu

2whitmesj@jmu.edu

Keywords: Google Earth, KML, JavaScript, geospatial, tectonics

\begin{abstract}
Google Earthis recognized as a highly effective visualization tool for geospatial information. However, there remain serious limitations that have hindered its acceptance as a tool for research and education in the geosciences. One significant limitation is the inability to translate or rotate geometrical elements on the Google Earth virtual globe. Here we present a new JavaScript web application to "Move and Rotate Google Earth Elements" (MaRGEE). MaRGEE includes tools to simplify, translate, and rotate elements, add intermediate steps to a transposition, and batch process multiple transpositions. The transposition algorithm uses spherical geometry calculations, such as the haversine formula, to accurately reposition groups of points, paths, and polygons on the Google Earth globe without distortion. Due to the imminent deprecation of the Google Earth API and browser plugin, MaRGEE uses a Google Maps interface to facilitate and illustrate the transpositions. However, the inherent spatial distortions that result from the Google Maps Web Mercator projection are not apparent once the transposed elements are saved as a KML file and opened in Google Earth.Potential applications of the MaRGEE toolkit include tectonic reconstructions, the movements of glaciers or thrust sheets, and time-basedanimations of other large-and small-scale geologic processes.
\end{abstract}

\section{Introduction}

In the years since its debut in 2005, the Google Earth virtual globehas become a highly effective visualization tool for geospatial information (Butler, 2006; Bailey and Chen, 2011). The initial wonder and awe of members of the general public at the ease with which they could fly to any place on the globe and view detailed imagery of the ground surface has been supplanted by its ubiquitous use as a geobrowser of Earth imagery and data. By integrating Keyhole MarkUp Language (KML), now an OpenGIS scripting language, within the Google Earth platform the utility of Google Earth was greatly expanded beyond just passive viewing of the modern-day Earth surface (Ballagh et al., 2011). Geoscientists and others have the means to display original data and models within a user-friendly, 3D virtual globe interface. Introduction of the TimeSpan feature further expanded the features of Google Earth to allow geoscientists to incorporate the $4^{\text {th }}$ dimension of time within Google 
Earthvisualizations. In many aspects, Google Earth has become the preeminent way to display 4D geoscientific and environmental information.

Unfortunately, there remain several limitations in Google Earth that have restricted its full potential as a research and educational tool. Many of these limitations have been previously documented in publications that describe workaround solutions (De Paor and Whitmeyer, 2011; Blenkinsop, 2012, Zhu et al., 2014b). Examples include Screen Overlays that must be coded outside of the Google Earth application (e.g. Whitmeyer, 2010; Dordevic, 2012), 3D COLLADA models that can be positioned on the Google Earth terrain, but must be created by an external application such as SketchUp (De Paor and Pinan-Llamas, 2006; De Paor and Williams, 2006; Dordevic et al., 2010; De Paor and Whitmeyer, 2011; Mochales and Blenkinsop, 2014), and an opaque ground surface that makes displaying and visualizing subsurface data and models challenging (De Paor et al., 2008; Whitmeyer and De Paor, 2008).

Perhaps the most significant limitation of Google Earth from a processfocused science perspective is the inability to transpose (translate, rotate) Google Earth elements, such as lines (paths), polygons, or groups of placemarks. This limitation makes it extremely difficult to show dynamic natural processes, as the creator of a visualization has to draft each incremental position of an element individually. Our initial solution to this problem used a command-line PERL script to load a KML file with geometrical components that the visualization creator wanted to translate or rotate (Whitmeyer and Patterson, 2013). Initially our application focused on local or regional transformations, and therefore didn't address aerial distortions of polygons or line elements that would occur when translating across multiple degrees of latitude (e.g. the methodology of Zhu et al., 2014a). However, as the focus of our Google Earth visualizations expanded to include movements and processes through geologic timescales (thousands to millions of years) it became apparent that our transposition algorithm needed to be based on spherical geometry to preserve the shapes and spatial relationships of Google Earth geometry elements across multiple degrees of latitude and longitude (e.g. De Paor, 1996).

Details of how geometry elements are represented in KML and processed in Google Earth have been documented elsewhere (Wernecke, 2009; Zhu et al., 2014a) and don't warrant reiteration here. In the sections that follow we focus onrelevant KML file structures, the importance of using spherical geometry to transpose Google Earth geometry elements, and the algorithms we use to accomplish spherical transpositions. This is followed by a description of the JavaScriptweb application: "Move and Rotate Google Earth Elements" (MaRGEE): http://geode.net/margee/. A github link to code for the files used in the MaRGEE web application and an example KML file that was produced using MaRGEE and the methods described beloware included in Appendix A.

\section{KML Structure}

MaRGEE functions by loading a KML or standard KMZ (zipped KML package) file that contains the Google Earth geometry elements (points, paths, polygons) to be transposed or edited (Fig. 1). The primitive KML geometry types (Google, 2013a; Fig. 2) that MaRGEE can handle are KmlPoint, KmlLineString (path) and 
92 KmlPolygon(polygon), as these proved to be most relevant in global-scale

93 transpositions of Google Earth elements.

94

95

96

97

98

99

100

101

102

103

104

105

106

107

108

109

110

111

112

113

114

115

116

117

118

119

120

121

122

123

124

125

126

127

128

129

130

131

132

133

134

135

136

137
Pathsare rendered in Google Earth assegments of a great circle. However, polygons are rendered in Google Earth by connecting individual coordinates, such that lines of constant bearing result in visually 'deformed' polygons near the poles (Fig. 3). By changing the KmlAltitudeMode in the polygon style element to something other than "Clamped..."polygons are rendered in the same manner as Paths. The same effect can be achieved by changing the polygon fill mode to "outline":KmlPolyStyle.setFill(0).

Other geometry types (KmlModel, KmlGroundOverlay,KmlMultiGeometry) were excludedfrom our implementation. KmlModel is used to display COLLADA models in Google Earth. It is anchored with a single coordinate (KmlLocation), scale (KmlScale) and model orientation (KmlOrientation). KmlGroundOverlay is positioned using KmlLatLonBox and is not particularly useful for rotation as it is bounded by lines of constant bearing. KmlMultiGeometry could be useful, as it represents all of the above as a collection of primitive geometries. However, it cannot be created directly in the Google Earth application, which significantly diminishes its usability in creating polygons and other KML elements.

MaRGEE can read and write to KML and KMZ filetypes (Google, 2013b). A KMZ file is a Zip archive that contains a KML document (doc.kml) and all of

theaffiliatedresources (imagery, COLLADA models, sound files. etc.) in an associated files folder. Users can browse local file structures and choose a file to manipulate, orloadfiles located on publicly accessible servers. The third party library used for file manipulationFileSaver.js caps the file size for uploads to 300MB. This KML file size would be equivalent to a single polygon containing about 8 million points.

Once a KML file is loaded and read by the browser as a text string (Mozilla, 2014), it is parsed using ge.parseKml (KMLString). This returnsa KMLObject, which is used in subsequentoperations. To allow users to choose which Google Earthelements will be subjected to manipulation,MaRGEE creates a list of KML elementssimilar to the "Places" menu in the stand-alone Google Earthapplication (upper left of Fig. 1).

\section{Calculations}

Operations that are possible in MaRGEE are: rotation, translation, inverse rotation, simplification, and editing of elements. In addition it is possible to create intermediary steps in a specified rotation or translation (Fig. 4), as well as assign aTimeSpan (KmlTimeSpan) to each step to facilitate time-based animation.

Our approach to transposing Google Earth elements uses spherical geometry, specifically the haversine (half-versed-sine) formula, as commonly used by navigators and astronomers (Sinnott, 1984). Rotation is calculated using the following sequence of formulas, where $\theta$ represents a clockwise rotation (inradians), $G$ is a geometry containing $n$ points $\left(P_{i}\right)$, and $E\left(\lambda_{E}, \varphi_{E}\right)$ isthe pole of rotation (Euler 
138 pole)with coordinates $\lambda_{i}, \varphi_{i}$ (longitude and latitude in radians). The firststep is to 139 calculate angular distance $c_{i}=\left|P_{i} E\right|$ :

$$
c_{i}=2 \operatorname{atan} 2\left(\sqrt{a_{i}}, \sqrt{1-a_{i}}\right)
$$

The next step is to find constant bearing $\rho_{i}$ between points $E$ and $P_{i}$ :

$$
\text { and } a_{i}=\sin ^{2}\left(\frac{\Delta \varphi}{2}\right)+\cos \varphi_{E} \cos \varphi_{i} \sin ^{2}\left(\frac{\Delta \lambda}{2}\right)
$$

$$
\text { where } \operatorname{atan} 2(x, y)=\frac{-x+\sqrt{x^{2}+y^{2}}}{y}
$$

$$
\rho_{i}=\operatorname{atan} 2\left(\sin (\Delta \lambda) \cos \varphi_{i}, \cos \varphi_{E} \sin \varphi_{i}-\sin \varphi_{E} \cos \varphi_{i} \cos (\Delta \lambda)\right)
$$

Finally, we find the coordinates of the destination point $P_{i}{ }^{\prime}$ given a starting point $E$, a distance $c_{i}$ and a bearing $\theta+\rho_{i}$ :

$$
\varphi_{i}^{\prime}=\arcsin \left(\sin \varphi_{E} \cos c_{i}+\cos \varphi_{E} \sin c_{i} \cos \left(\theta+\rho_{i}\right)\right)
$$

$$
\lambda_{i}^{\prime}=\lambda_{i}+\operatorname{atan} 2\left(\sin \left(\theta+\rho_{i}\right) \sin c_{i} \cos \varphi_{E}, \cos \left(\theta+\rho_{i}\right)-\sin \varphi_{E} \sin \varphi_{i}^{\prime}\right)
$$

This procedure, when iterated over all $n$ points, will produce a rotated geometry $G^{\prime}$.

Translation performed on a sphere can berepresented by a rotation around a particular Euler pole. To translate geometry $G$ an angular distance $d$ along a bearing $\theta$ we first determine the destination point $E$ that is $\pi$ radians away from $P_{i}$ along a bearing of $\theta+\pi / 2$. Then, we perform a rotation, as described above, of point $P_{i}$ around $E$ for $d$ radians. The JavaScriptimplementation used for thetransposition of coordinates in both the rotation and translationfunctions is from the thirdparty library:geo.js (Veness, 2014).

\section{Determining the Euler Pole and Angle of Rotation for a Transposition}

TheInterpolate Rotation Parametersfunction gives the location of the Euler pole $E$ and the angle of rotation $\theta$ if two geometries $G$ and $G^{\prime}$ are provided, where the geometries contain at least two analogous points. In other words, given two locations of the same polygon, the interpolation function finds the Euler pole and angle of rotation that would enable the transposition between the starting and ending locations. The first step is to find midpoint pairs $M_{i}$ along $P_{i} P_{i}{ }^{\prime}$ and $M_{j}$ along $P_{j} P_{j}{ }^{\prime}$. Next, we find the bearings $\theta_{i}$ for $P_{i} M_{i}$ and $\theta_{j}$ for $P_{j} M_{j}$, and then calculate the intersection point $E$ between the two great circles defined with starting point $M_{i}, M_{j}$ and bearings $\theta_{i}+\pi / 2, \theta_{j}+\pi / 2$. The angular distance $P_{i} P_{i}{ }^{\prime}$ is the angle of rotation $\theta$. As there are two possible solutions for the pole, $E$ and antipodal $E$ " there are also 
174 two possible angles of rotation. Our convention is that the first geometry $G$ is the 175 geometry prior to transformation, and the second geometry selected $\left(G^{\prime}\right)$ is post

176 rotation. Thus, the first solution presented is the counter-clockwise rotation,

177 followed by the clockwise rotation solution. Our JavaScript routine for the

178 Interpolate Rotation Parametersfunction is as follows:

179

180

181

182

183

184

185

186

187

188

189

190

191

192

193

194

195

196

197

198

199

200

201

202

203

204

205

206

207

208

209

210

211

212

213

214

215

216

217

218

219

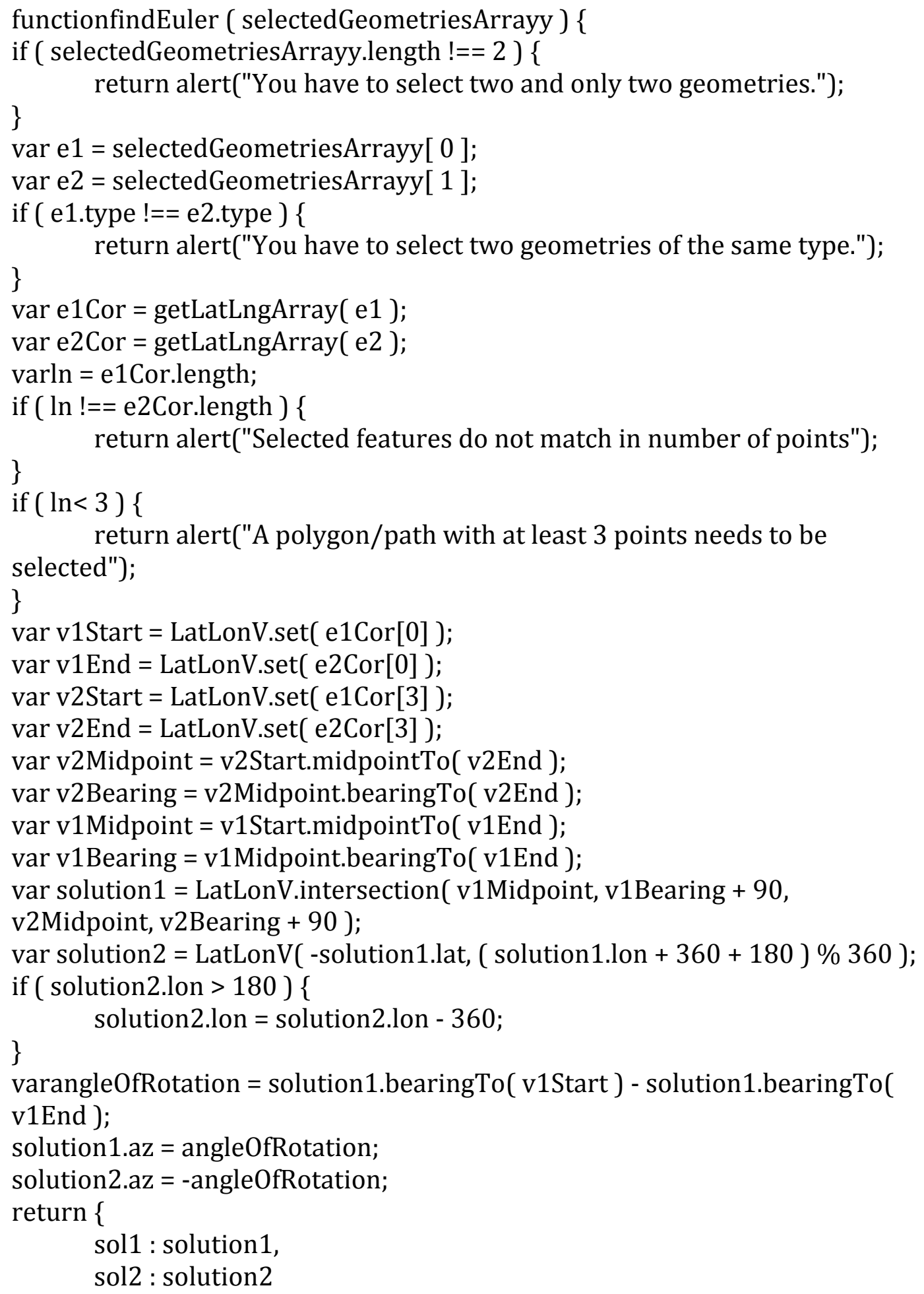


\};

\}

Note that we check to see that the user has selected two elements (geometries) and that the elements have the same number of vertices (points). This is to verify that the two elements are, in fact, a single element in its original and transposed positions.

\section{Simplification of Paths and Polygon Outlines}

The simplification routine is included within MaRGEE in order to reduce calculation times for transposing elements and improve the smoothness of time-based visualizations of elements in Google Earth. Early versions of MaRGEE showed that detailed polygons with thousands of points took several seconds to transpose. If the Google Earth viewpoint is at a distance where the full globe is visible, most polygons don't need to be more detailed than a few hundred points, and this routine automates the process of simplifying the polygons without significantly changing the apparent resolution of the element. The simplification routine reduces the number of points in a geometry based upon the parameter $\epsilon$ used in the Stack-based line simplification routineof Douglas and Peucker(1973). It recursively splits a geometry starting from the ends, finding the furthest point from a line connecting start and end points. If this distance is smaller than $\epsilon$, the point is discarded.

Otherwise, the whole process repeats until sub geometries become lines.The JavaScript routine for simplification is derived from the third party library: douglas peucker line simplification.js(Robinson, 2010).

\section{Web Interface and Workflow}

TheMaRGEE web interface is divided into three sections: the main window that shows the Google Earth virtual globe,a panel of tools on the left, and several drop down menus at the top (Fig. 1). The tools panel is subdivided into nine collapsible subsections, where clicking on the minus icon will expand or collapse the subsection content. The subsections include: KML list, Simplify, Translate, Rotate, Intermediate Steps, and Batch Processing (see Figs. 1, 4, 6).The top panel includes the dropdown menus: File (upload and download KML and KMZ files), Edit (Undo and Redo options), Map Options (toggle visibility of a latitude / longitude grid), Tools (includes a tool to Interpolate Rotation Parameters - i.e. find the pole of rotation for a transposed polygon), and View Panels (makehidden tools panels reappear); see Table 1.

The first step in operating MaRGEE is to load a KML file that contains Google Earth elements (points, lines, polygons) to be transposed. This can be done by simply dragging and dropping a KML file at any place in the MaRGEE window, or by clicking the File dropdown menu and browsing for the desired KML or KMZfile. The File menuis also where you select the option to save your transposed elements as KML or KMZ files. 
Once a KML or KMZ file is loaded, its content is listed in the KML List section in the tools panel along the right side of the MaRGEE window. Each KML elementwithin the file will be listed by name, as derived from the <name $>$ associated with each $<$ Placemark $>$ header. Here you can select individual KML elements or groups of KMLelements to manipulate by lassoinga sequential group or clicking on individual elements while holding Ctrl/Command. Selected elements and their intermediate steps can also be deleted by clicking the trashcan icon, or hidden from view by clicking the "eye"

As described above, the Simplify tool smoothsthe path or polygon outline and reduces the number of vertices in selected KML elements.This is useful when a given polygon has thousands of vertices, but will typically be viewed from a global viewpoint. An example of this is India, as shown in Fig. 1.

The Translate toolmoves selected KML elements by allowingusers to set the initial heading of translation in decimal degrees measured in a clockwise direction from north ( 0 degrees), and the distance of the translation in $\mathrm{km}$. Alternatively, the user can drag the red and white bullseyemarkerto a new location on the map, and the initial heading and distance will be shown in their respective fields. Clicking the Translate button will then move the element(s).

Rotation is performed in similar manner to translation. Once KML elements are selected, the user entersan Euler pole latitude and longitude in decimal degrees, followed by the angle of rotation in degrees (clockwise is positive). Location of the Euler pole can be manually positioned by dragging the rotation marker(red circle with arrows)on the map. Note that a user cancalculate the position of an Euler pole and the angle of rotation if two elements are selected that are the result of one consecutive rotation. This is accomplished by clicking Interpolate Rotation Parameters in the Tools dropdown menu. Two antipodal solutions will appear at the top of the Rotate subsection.

When building animations of KML elements that move though time (e.g. tectonic reconstructions) we found it useful to have the option to interpolate intermediate steps between a starting and ending locations. This is accomplished with the Intermediate Steps tool, whichbreaks up a given rotation or translation, as defined above, and adds a specified number of intermediate steps (Fig. 4). Note that the number of steps defaults to 0 , which results in no intermediate steps. Users can also create a Google EarthTimeSpan animation (Google, 2013c) by selecting the Create Time Span box. The Start and Stop fields assign the beginning and ending dates for the Time Span animation. We typically use integer numbers to represent millions of years ago (mya), since Google Earth can't handle Time Span dates greater than a few thousand years.

If many transpositions are desired the user can process all of the transpositions as a single batch, using the Batch processing tool. This tool will accept a string of commands with one or more lines processed in sequence, where each line is a single 
311 transposition. Each command line starts by specifying the transposition type: a " $\mathrm{t}$ " 312 for translate, and an " $r$ " for rotate. The syntax for the command line for a rotation 313 continues with values for Euler pole latitude and Euler pole longitude in decimal 314 degrees, followed by the rotation angle: " $r-37.020 .0-90$ ". The syntax for a translation in meters, and looks like:" $t 1208000$ "By adding three extra arguments to the command lines for either of the operations the user can set the number of intermediary steps andthe start and stop time for a Time Span. For example: " $r$-37.0 20.0 -90 506 "will rotate the selected elements around an Euler pole located at -37.0 degrees latitude and 20.0 degrees longitude for 90 degrees in a counterclockwise direction and create five intermediate steps (seven figures in total) with a time span from 0 to 6 mya (see the India example in Fig. 4).

Batch processing is especially helpful for tectonic reconstructions where stage pole rotational information is known for a set of time slices. For example, the following sequence of command lines when entered in the batch processing field will produce a Google Earth Time Span animation of the movement of the India subcontinent relative to Eurasia over the past 80 million years (data from Copley et al., 2010).

$\begin{array}{rlrllll}r & 26.61 & 23.39 & 2.60 & 5 & -1 & 4 \\ r & 25.41 & 20.11 & 2.10 & 5 & 4 & 9 \\ r & 23.81 & 21.06 & 2.56 & 5 & 9 & 14 \\ r & 24.29 & 20.88 & 2.85 & 5 & 14 & 19 \\ r & 21.36 & 27.95 & 3.65 & 5 & 19 & 24 \\ r & 23.39 & 27.74 & 3.21 & 5 & 24 & 29 \\ r & 24.43 & 27.68 & 3.25 & 5 & 29 & 34 \\ r & 22.92 & 29.37 & 4.15 & 5 & 34 & 39 \\ r & 21.74 & 28.84 & 4.08 & 5 & 39 & 44 \\ r & 20.35 & 25.68 & 3.94 & 5 & 44 & 49 \\ r & 21.67 & 19.67 & 6.34 & 5 & 49 & 54 \\ r & -19.89 & -161.39 & -7.36 & 5 & 54 & 59 \\ r & -18.79 & -163.25 & -7.30 & 5 & 59 & 64 \\ r & -20.47 & -168.60 & -4.54 & 5 & 64 & 69 \\ r & -21.06 & -169.94 & -4.14 & 5 & 69 & 74 \\ r & 20.79 & 9.56 & 4.39 & 5 & 74 & 79\end{array}$

See the online supplement for the KML file (GreaterIndiaMovement.kml) of the animation produced by this batch.

\section{Challenges for Implementation}

\section{Polygon Fill}

During trouble-shooting phases in the development of the MaRGEE tool we encountered several issues that largely derived from quirks in the Google Earth 
application. One of the challenges we encountered was polygons that filledincorrectly (e.g. the fill occurredoutside the polygon's outline; Fig. 5a) when the polygon straddledthe meridian or antimeridian. When a polygon'slongitudinal coordinates are within the range of -180 to 180 ,Google Earth's polygon filling routine renders incorrectly when successive vertices fall on opposite sides of the antimeridian (orange polygon in Fig. 5a). However, in Fig. 5b the yellow polygon is rendered correctly across the antimeridianbecause the longitudes have been normalized to a range of 0 to 360 by adding 180 to the real longitude value. A similar rendering error can happen when a polygon intersects the meridian, but in this situation polygons are correctly rendered when longitudes are between the normal range of -180 to 180 .

To remedy this problem, we presume that no polygon would span more than 180 degrees in longitude. Next, after every transformation and normalization to -180 to 180 longitude we check to see if the difference between the maximal longitude and the minimal longitude is smaller than 180 . If so, we continue with this normalization, otherwise wenormalize the longitudes to a range of $0-360$.

As mentioned above, polygon fill is determined by lines of constant bearing connecting vertices. Whena polygon is transposed close to poles or around poles, rendering can significantlydistort the shape of a polygon (Fig. 3) or render the polygon fill outside of the polygon outline (Fig. 5a). Though not an ideal solution, our approach is to look for three problematic situations after each transposition: 1 . isthe maximum latitude in a polygon greater than 85 degrees, 2.is the minimumlatitude smaller than -85 degrees, or 3 . dothe maximum and minimum longitudes span more than 180 degrees. If any of these conditions are met, we set the outline color of the polygon to the same value as the fill color and change the style of the polygon to "outline" only. When these conditions no longer apply the original style of the polygon("filled") is restored. This way, the user sees either a filled or an outlined polygon at any given position.

\section{Calculation Speed}

When we tested the transposition algorithmwith the Google Earth plugin we noticed significant increases in processing timewhentransposing geometries with many vertices. For example, a KML file with a single polygon containing $\sim 40,000$ vertices was rotated 45 degrees around an Euler pole located at longitude $45^{\circ}$ and latitude $45^{\circ}$. The test machine was an Intel i7 3 rd generation cpu at $2.66 \mathrm{GHz}$, running Windows 7 Professional and Google Chrome browser (Version 38.0.2125.101). After performing the first run, which took a relatively lengthy $14 \mathrm{~s}$, we started a timing procedure to find the bottleneck. The procedure we used was as follows:

1. loop through all polygon coordinates and extract them to JavaScript arrays "lat" and "lon"

2. split arrays amongst four webworkers

3. workers perform operations necessary for rotation

384

4. each worker sends data to main script 
5. transformed coordinates are merged to a single array "latNew, lonNew"

6. latNew and lonNew are pushed to new polygon coordinates The timing for each step showed that about $95 \%$ of the time was spent getting and putting coordinates from KmlPolygon. It turns out that it is much faster to do getKml() on KmlPlacemark with KmlPolygon geometry to export theKML string and from this string to parse the coordinates. The inverted procedure is done to calculate new coordinates for theGoogle Earth polygon. With this new procedure, the total time necessary for the benchmark operation was reduced to 1.1s, using the same hardware.

\section{Deprecation of the Google Earth API and the Google Maps interface}

The MaRGEE tool was originally implemented using the Google Earth Application Program Interface (API) - e.g. the web browser plugin (cf Zhu et al., 2014a). However, due to increasing incompatibility of the Google Earth API with modern web browsers, Google has deprecated the Google Earth API and it will cease to be available after 12/15/2015 (Google, 2013d). Our Google Earth API implementation will remain functional until that date and can be accessed here: http://dev.geode.net/poly/ However, given the limited future of the Google Earth API, we have rewritten MaRGEE using the Google Maps web interface: http://dev.geode.net/poly-maps/

In many ways the Google Maps implementation is superior to the previous Google Earthplugin platform. Google Maps does notrequire installation and use of a browser plugin, which makes it more user friendly and available across various platforms. In addition, the MaRGEE tools work significantly faster with Google Maps than the Google Earth plugin implementation. One complication with Google Maps is that it uses theWGS 84 /Pseudo-Mercator projection to display maps (EPSG, 2014), as opposed to a virtual globe. Google Maps uses this projection to preserve angles for street-level viewing, which is how the majority of users use Google Maps. However, when viewing Google Maps at the global scale, significant distortion occurs at high latitudes (Fig. 6a). In addition, the Google Maps interface does not wrap around at the poles, so polygons that straddle polar regions look quite peculiar.However, this is just an artifact of the Google Maps projection, as the polygons render correctly and are not distorted when imported into Google Earth (Fig. 6b).

\section{Conclusions}

The limitations of Google Earth that restrict its use as a serious research tool have been known forseveral years, and many of these limitations still remain. Developers within the geoscience and cyber communities have had to create work-around solutions to ameliorate many of these deficiencies. This paper documents one approach to address the issue of moving KML elements across the Google Earth globe. The MaRGEEweb application presented here (http://geode.net/margee/) provides a user-friendly GUI for accurate spherical transpositions at global scales. By adding TimeSpan information to transpositions, the fourth dimension of time can be included to create animations of movements and processes for Google Earth. We 
431 envision geoscientists and others using this tool for plate tectonic reconstructions, 432 animations of continent and micro-continent movements through time (see

433 Appendix A), and illustrations of other large-scale geologic processes. However, the 434 MaRGEE toolkit is equally adept at regional and local transpositions, and should

435 therefore aid in a wide variety of applications that require moving things around the

436 Google Earth virtual globe, such as landslides or the movements of glaciers or thrust

437 sheets.Planned future developments for this toolkit include the ability toalter or

438 deform polygons, though the algorithms and inherent assumptions for these

439 transformations are more complex.

440 The deprecation of the Google Earth API and browser plugin resulted in our

441 use of Google Maps as the primary interface for the MaRGEE toolkit,which has both

442 advantages (speed of processing) and disadvantages (visual distortionthat is most

443 apparent near the poles). Apparent distortion in the Google Maps Web Mercator

444 projection has little effect for small polygons (meters to kilometers in scale)

445 transposed short distances. For transpositions of larger polygons across greater

446 distancesan extra verification step may be required to make sure elements are in

447 the desired locations and/or orientations in Google Earth after transposition.

448 However, as Google Earth is a virtual globe and does not incorporate the distortion

449 of the Web Mercator projection, all transposed KML elements appear correctly in

450 the stand-alone application.We plan to update the browser platform for MaRGEE to

451 whatever Google releases as an update to the Google Earthbrowser plugin, but at

452 present we are not aware of the timeframe from Google for the release of a new

453 web-based version of Google Earth.

454

455

\section{Acknowledgements}

456 This research was supported, in part, by the National Science Foundation (DUE

$457 \quad \# 1323468$ ) and a Google Curriculum Proposal. The authors thank Declan De

458 Paorand two anonymous reviewers for helpful comments and suggestions.

459

460

461

462

463

464

465

466

\section{Appendix A. Supporting files}

Code for all of the files used in the MaRGEE web application is available at the github URL: https://github.com/mladen-dordevic/margee

The online supplement includes the file GreaterIndiaMovement.kml, which is a TimeSpan animation of the relative movement of the India subcontinent relative to Eurasia over the past 80 million years. 
References

468 Bailey, J.E.,Chen,A.,2011.Theroleofvirtualglobesingeoscience.Comput.Geosci.

Ballagh, L.M.,Raup,B.H.,Duerr,R.E.,Khalsa,S.J.S.,Helm,C.,Fowler,D.,Gupte,A., 2011.Representingscientific datasetsinKML:methodsandchallenges. Comput. Geosci.37(1),57-64.

Blenkinsop, T.G.,2012.Visualizingstructuralgeology:fromExceltoGoogleEarth. Comput. Geosci.45(1),52-56.

Butler, D.,2006.Virtualglobes:theweb-wideworld.Nature439(7078),776-778.

Copley, A., Avouac, J.-P., and Royer, J.-Y.,2010. India-Asia collision and the Cenozoic slowdown of the Indian plate:Implications for the forces driving plate motions, J.Geophy. Res. 115, B03410, doi:10.1029/2009JB006634.

De Paor, D.G., 1996. Computation of orientations for GIS - the 'Roll' of quaternions.Comput. MethodsGeosci.15,447-456.

De Paor,D.G.,Pinan-Llamas,A.,2006.Applicationofnovelpresentationtechniques to astructuralandmetamorphicmapofthePampeanOrogenicBelt,NW Argentina. Geol.Soc.Am.Abstr.Programs38(7),326.

De Paor,D.G.,Williams,N.R.,2006.Solidmodelingofmomenttensorsolutionsand temporalaftershocksequencesfortheKiholoBayearthquakeusingGoogle Earth withasurfacebump-out.EosTrans.Am.Geophys.Union,87;(abstract S53E-05).

De Paor, D.G., Whitmeyer, S.J., Gobert, J., 2008. Emergent Models for Teaching Geology and Geophysics Using Google Earth, EosTrans.Am.Geophys.Union, 89 (53)(abstract ED31A-0599).

De Paor,D.G.,Whitmeyer,S.J.,2011.Geologicalandgeophysicalmodelingonvirtual globes usingKML,COLLADA,andJavascript.Comput.Geosci.37(1),100-110.

Dordevic, M.M., De Paor, D.G., Whitmeyer, S.J., Beebe, M.R., 2010. Animated COLLADA Models and Virtual Field Trips Featuring Volcanism in Various Tectonic Settings on Planet Earth and Other Rocky Planets and Moons.Geol.Soc.Am.Abstr.Programs42 (5), 420.

Dordevic, M.M.,2012.Designinginteractivescreenoverlaystoenhance effectiveness ofGoogleEarthgeoscienceresources.Geo.Soc.Am.SpecialPap.492, 105-111.

Douglas, D.H,Peucker, T.K., 1973. Algorithms for the reduction of the number of points required to represent a digitized line or its caricature. Cartographica: The International Journal for Geographic Information and Geovisualization 10.2, 112- 
EPSG, 2014. EPSG:3857 Projected coordinate system.

516 (http://epsg.io/3857)(accessed 1/27/2015).

517

518 Google, 2013a.Keyhole Markup Language - KML Documentation

519 Introduction.(https://developers.google.com/kml/documentation/)

520 (accessed1/25/2015).

521

522

523

524

525

526

527

528

529

530

531

532

533

534

535

536

537

538

539

540

541

542

543

544

545

546

547

548

549

550

551

552

553

554

555

556

557

Google, 2013b.Keyhole Markup Language - KMZ Files.

(https://developers.google.com/kml/documentation/kmzarchives) (accessed $1 / 25 / 2015)$.

Google, 2013c.Keyhole Markup Language - TimeSpan.

(https://developers.google.com/kml/documentation/kmlreference\#timespan)

(accessed 1/25/2015).

Google, 2013d.Deprecation of Google Earth API.

(http://googlegeodevelopers.blogspot.com/2014/12/announcing-deprecation-ofgoogle-earth.html) (accessed 1/25/2015).

Mochales, T. and Blenkinsop, T.G., 2014. Representation of paleomagnetic data in virtual globes: A case study from the Pyrenees.Comput. Geosci. 70, 56-62.

Mozilla, 2014.Mozilla Developer Network - Web API Interfaces -

FileReader.readAsText. (https://developer.mozilla.org/en-

US/docs/Web/API/FileReader.readAsText) (accessed 1/25/2015).

Robinson, G.J., 2010. Douglas Peucker line simplification.

(https://gist.github.com/zeke/543273) (accessed 1/27/2015).

Sinott, R.W., 1984. Virtues of theHaversine.Sky \& Telescope (68), 159.

Veness, C., 2014. Movable Type Scripts - Calculate distance, bearing and more between Latitude/Longitude points. (http://www.movable-

type.co.uk/scripts/latlong.html) (accessed 12/8/2014).

Whitmeyer, S.J., De Paor, D.G., 2008. Large-scale Emergent Cross Sections of Crustal Structures in Google Earth.Geol.Soc.Am.Abstr.Programs 40 (6), 189.

Whitmeyer, S.J., 2010.Creating Advanced Elements for Interactive Geologic Maps in Google Earth Using an HTML-based Toolkit.Geol.Soc.Am.Abstr.Programs42 (5), 421.

Whitmeyer, S., Patterson, C.R., 2013. Building tectonic reconstructions in Google Earth using a new toolkit to move and rotate polygons.

558 Geol.Soc.Am.Abstr.Programs45 (1), 109. 
560 Zhu, L.,Wang,X., Pan, X.,2014a. Moving KML geometry elements within Google

561 Earth.Comput.Geosci.72(1), 176-183.

562

563 Zhu, L.,Wang,X.,Zhang,B.,2014b.Modelingandvisualizingboreholeinformation on

564 virtualglobesusingKML.Comput.Geosci.62(1),62-70.

565

566 


\section{Figure and Table Captions}

568

569

570

571

572

573

574

575

576

577

578

579

580

581

582

583

584

585

586

587

588

589

590

591

592

593

594

595

596

597

598

599

600

601

602

603
Figure 1. View of the initial MaRGEE screen after a KML file has been loaded. The file consists of three elements: a filled polygonnamed India, an outlined polygon named India Outline, and a filled polygon named Sri Lanka. The India polygon is selected, and the India Outline and Sri Lanka polygons have been hidden from view.

Figure 2. Schematic of geometry types in KML file structure.

Figure 3. Rendering of pathsversus polygons. Each square has green outlines drawn as paths and red outlines around blue polygons; note the deviation from a uniform square in the polygon rendering as the object are positioned closer to the pole.

Translation of geometry with 10 intermediary steps along 18 degrees bearing along a distance of $18000 \mathrm{~km}$.

Figure 4. MaRGEE screen illustrating the Rotate and Intermediate Steps tools. India is rotated -90 degrees (counterclockwise) around a pole of latitude $-37.0^{\circ}$ and longitude $20.0^{\circ}$. The number of intermediate steps is 5 , resulting in 7 incremental polygons of India, including the starting and ending polygons. The Time Span box is also selected, which will produce a KML file that activates the Google EarthTimeSpan animation box to incrementally show the movement of India from 6 (mya) to 0 (mya).

Figure 5. Antimeridian rendering problem where a polygon straddles the antimeridian; A. orange polygon fill is incorrectly drawn outside the polygon outline, B. yellow polygon fill is correctly drawn within the polygon outline.

Figure 6. A. MaRGEE screen showing an incremental translation of the India polygon to the north, where the distortion from Google Maps's Mercator projection becomes increasingly manifest. B. Google Earth screen showing the same polygons rendered correctly on the virtual globe.

Table 1. Dropdown menus available at the top of the MaRGEE screen (first column), with the available options for each menu (second column). 
E MaRGEE Flle - Edit - Map Options - Tools - View Panels -

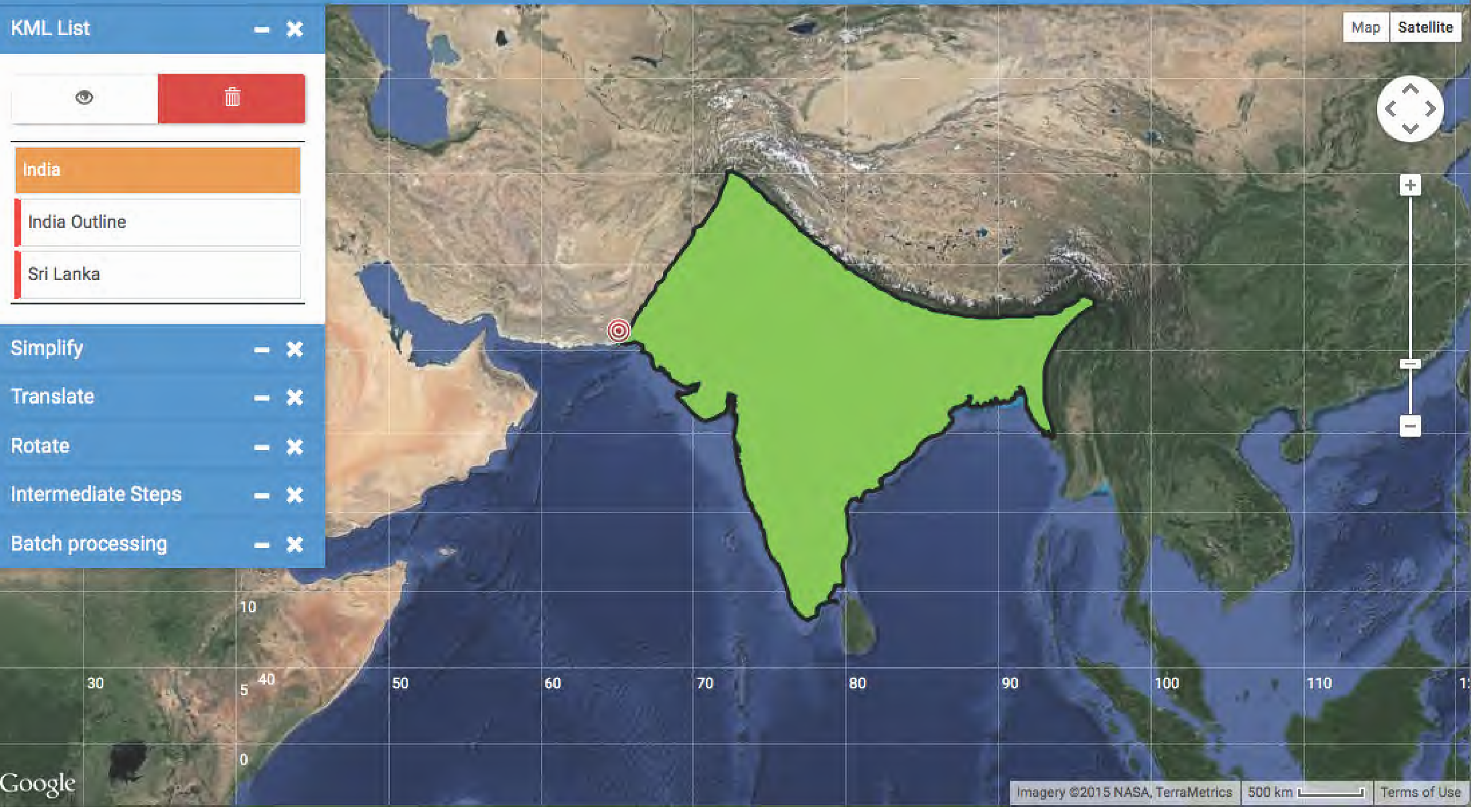




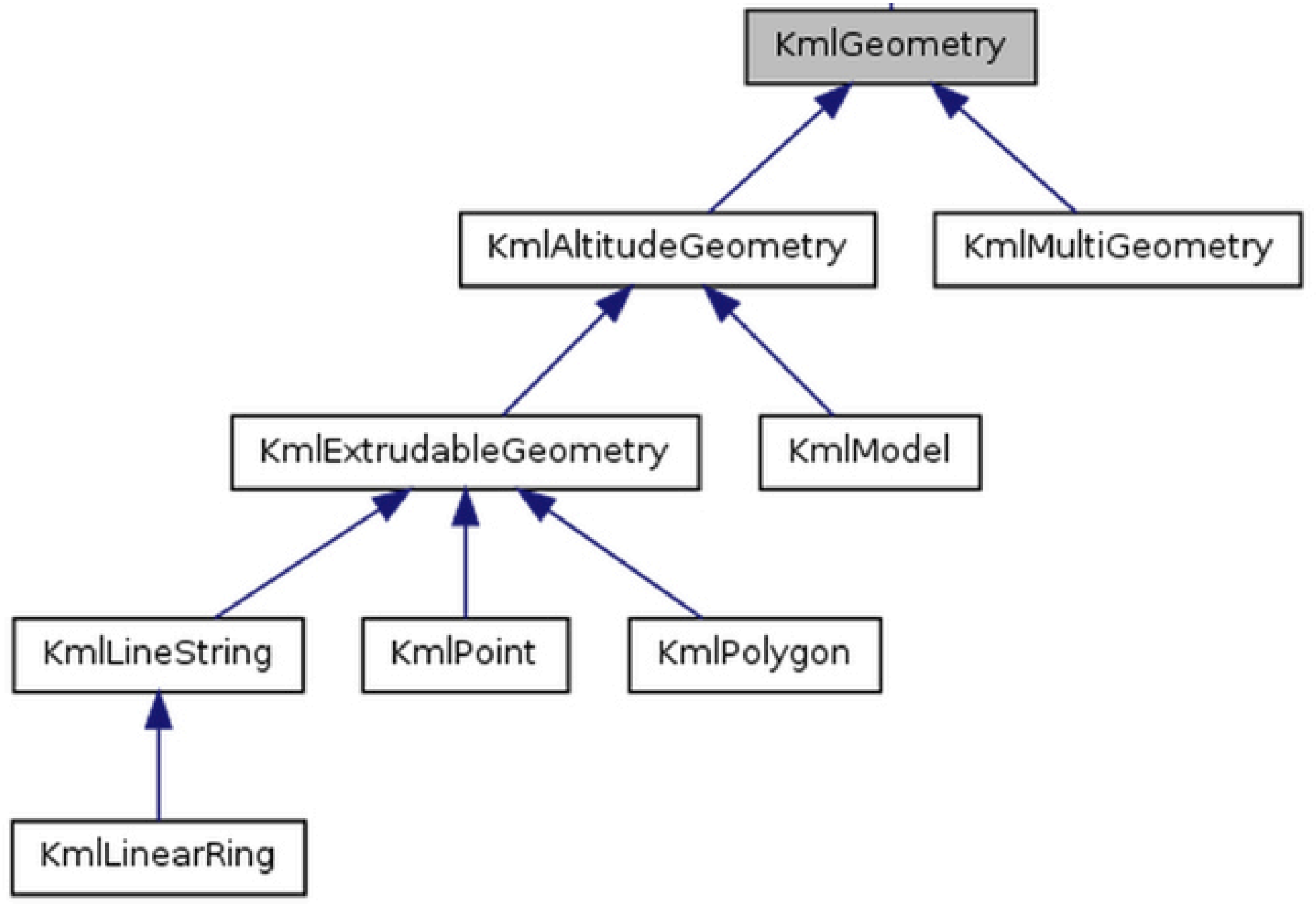




\section{Figure 3}

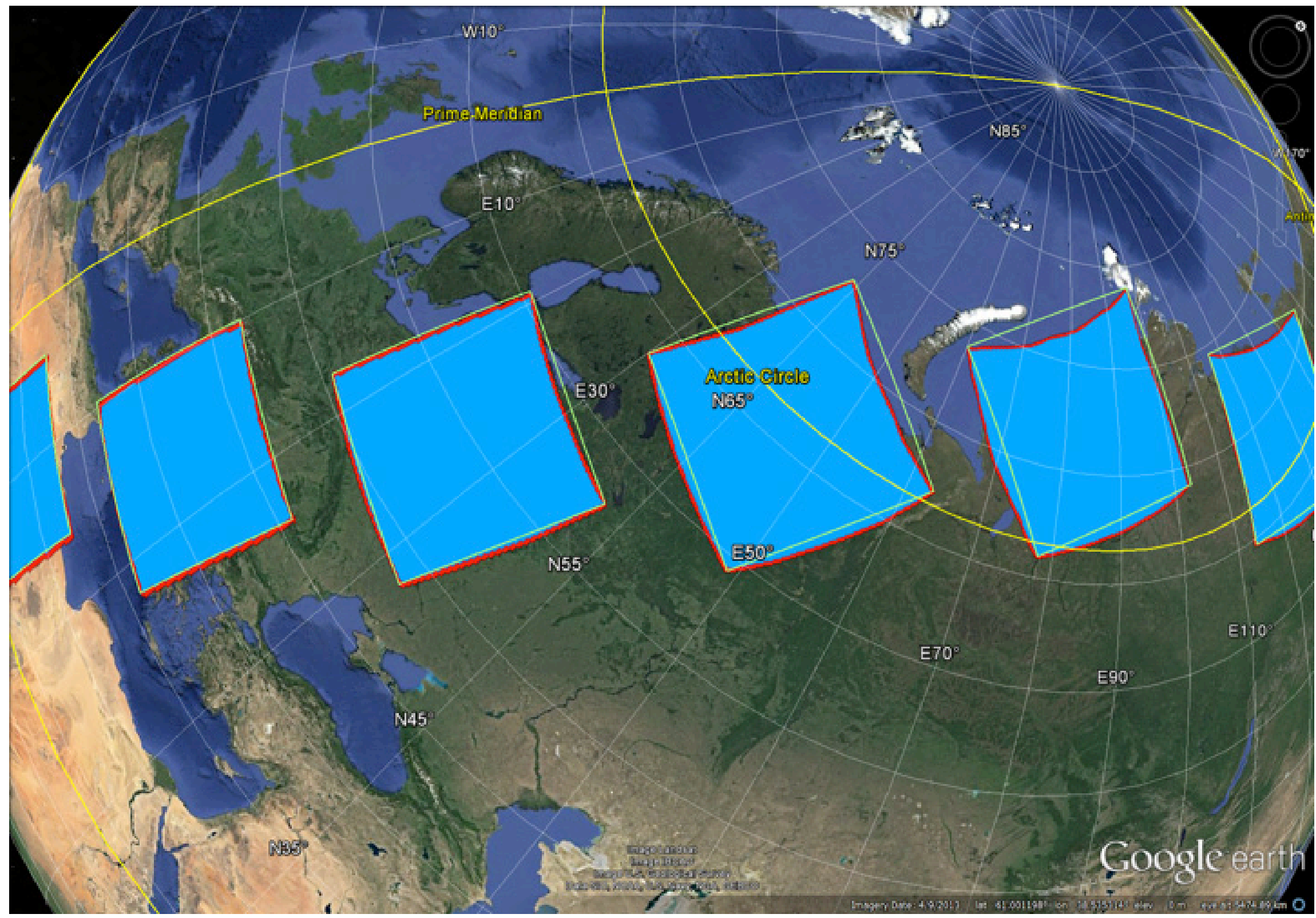




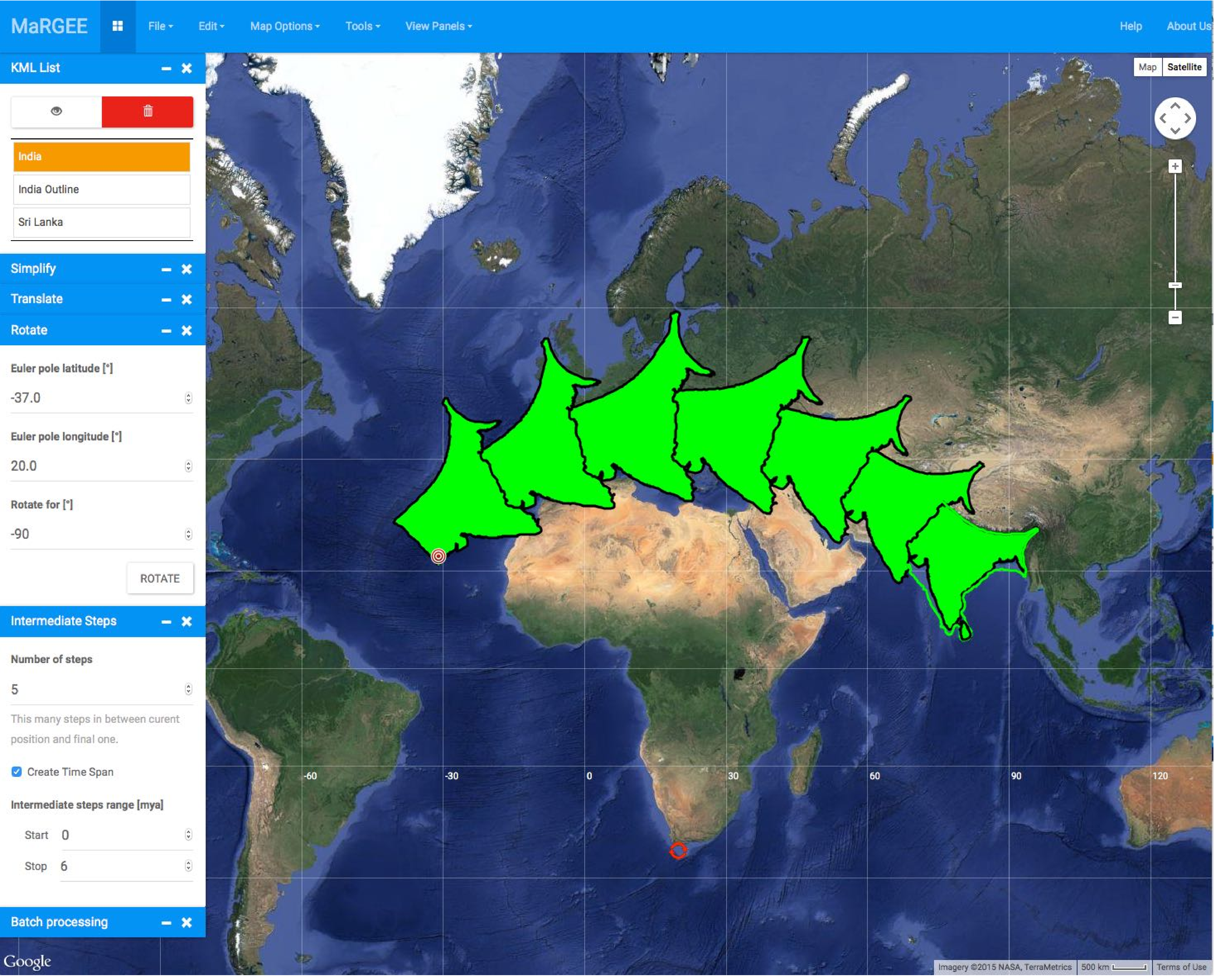


Figure 5

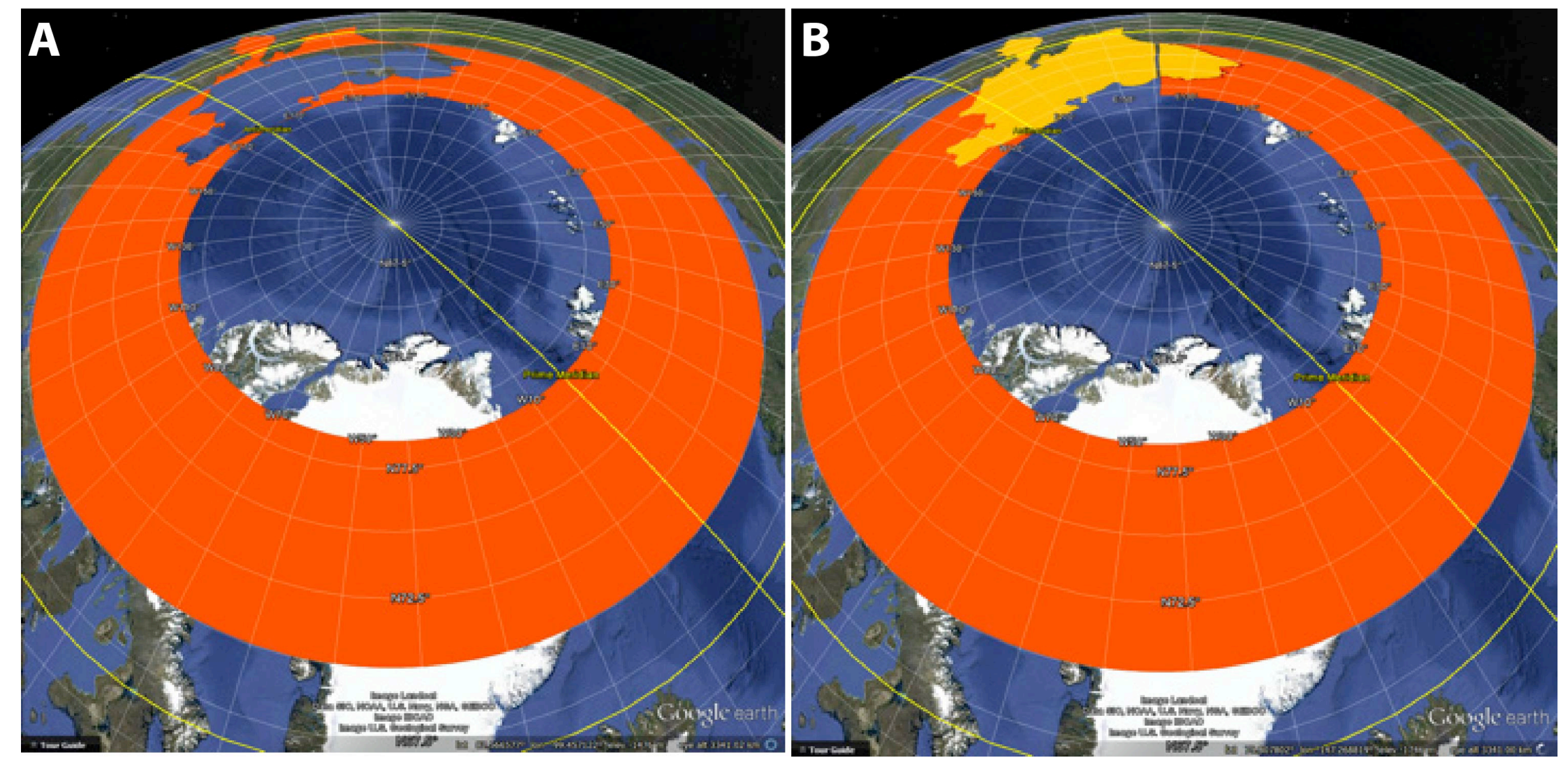




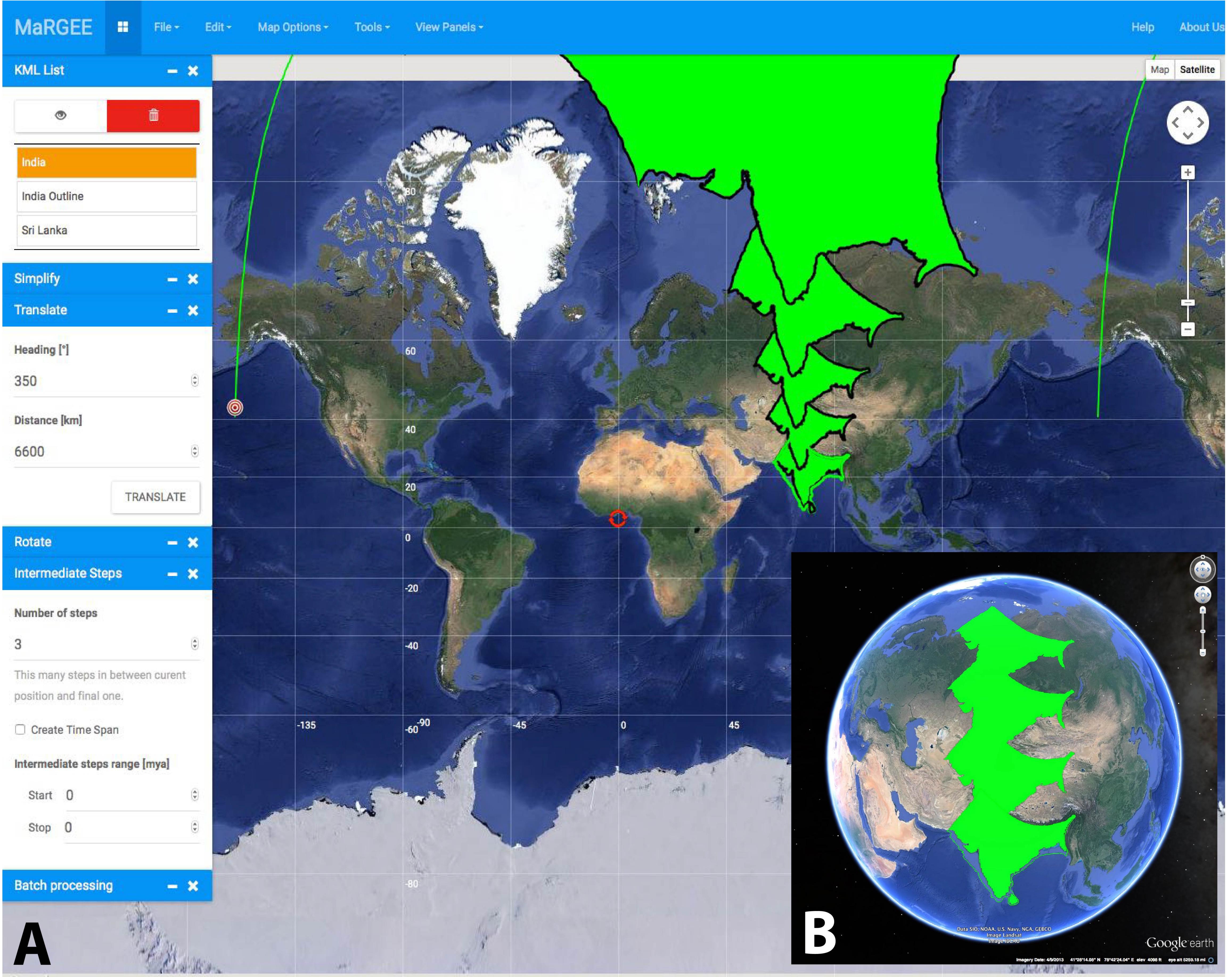

